BMJ Open

Diabetes

Research

\& Care

\title{
Evaluation and management of COVID- 19-related severity in people with type 2 diabetes
}

\author{
Bowen Wang (D) ,,2 Benjamin S Glicksberg, ${ }^{3,4}$ Girish N Nadkarni, ${ }^{4,5}$ \\ Deepak Vashishth ${ }^{1,2}$
}

To cite: Wang B, Glicksberg BS, Nadkarni GN, et al. Evaluation and management of COVID19-related severity in people with type 2 diabetes. BMJ Open Diab Res Care 2021;9:e002299. doi:10.1136/ bmjdrc-2021-002299

- Additional supplemental material is published online only. To view, please visit the journal online (http://dx.doi. org/10.1136/bmjdrc-2021002299).

Received 31 March 2021 Accepted 25 August 2021

Check for updates

(c) Author(s) (or their employer(s)) 2021. Re-use permitted under CC BY-NC. No commercial re-use. See rights and permissions. Published by BMJ.

For numbered affiliations see end of article.

Correspondence to Dr Deepak Vashishth; vashid@rpi.edu

\section{ABSTRACT}

Introduction People with type 2 diabetes (T2D) have an increased rate of hospitalization and mortality related to COVID-19. To identify ahead of time those who are at risk of developing severe diseases and potentially in need of intensive care, we investigated the independent associations between longitudinal glycated hemoglobin (HbA1c), the impact of common medications (metformin, insulin, ACE inhibitors (ACEls), angiotensin receptor blockers (ARBs), and corticosteroids) and COVID-19 severity in people with T2D.

Research design and methods Retrospective cohort study was conducted using deidentified claims and electronic health record data from the OptumLabs Data Warehouse across the USA between January 2017 and November 2020, including 16504 individuals with T2D and COVID-19. A univariate model and a multivariate model were applied to evaluate the association between 2 and 3-year HbA1c average, medication use between COVID-19 diagnosis and intensive care unit admission (if applicable), and risk of intensive care related to COVID-19.

Results With covariates adjusted, the HR of longitudinal $\mathrm{HbA} 1 \mathrm{c}$ for risk of intensive care was 1.12 (per $1 \%$ increase, $p<0.001$ ) and 1.48 (comparing group with poor $(\mathrm{HbA} 1 \mathrm{c} \geq 9 \%)$ and adequate glycemic control $(\mathrm{HbA1c}$ $6 \%-9 \%), p<0.001)$. The use of corticosteroids and the combined use of insulin and metformin were associated with significant reduction of intensive care risk, while ACEls and ARBs were not associated with reduced risk of intensive care.

Conclusions Two to three-year longitudinal glycemic level is independently associated with COVID-19-related severity in people with T2D. Here, we present a potential method to use $\mathrm{HbA1c}$ history, which presented a stronger association with COVID-19 severity than single-point HbA1c, to identify in advance those more at risk of intensive care due to COVID-19 in the T2D population. The combined use of metformin and insulin and the use of corticosteroids might be significant to prevent patients with $\mathrm{T} 2 \mathrm{D}$ from becoming critically ill from COVID-19.

\section{INTRODUCTION}

COVID-19 has become a global pandemic causing 4.5 million deaths worldwide as of 30 August 2021. ${ }^{1}$ With slow vaccination rate and spread of new variants, this number is expected to rise. $^{23}$ Individuals with type 2 diabetes (T2D) have been identified to

\section{Significance of this study}

What is already known about this subject?

- People with type 2 diabetes have an increased rate of hospitalization and mortality related to COVID-19.

What are the new findings?

- Two to three-year longitudinal glycemic control is most significantly associated with COVID-19-related severity in people with type 2 diabetes.

- The combined use of metformin and insulin and the use of corticosteroids are significantly associated with a reduction of intensive care risk in people with type 2 diabetes.

- The use of antihypertensive medications among people with type 2 diabetes is not associated with decreased risk of intensive care.

How might these results change the focus of research or clinical practice?

- The study emphasizes the importance of proper management glycemic level over longer period in reducing the risk of developing severe diseases from COVID-19. The method using HbA1c history could allow for personalized assessment and management of subsequent care related to COVID-19 in advance.

be a high-risk group for both the infection and development of severe disease of COVID-19. ${ }^{4-8}$ It is demonstrated that people with T2D have an increased rate of hospitalization and mortality related to COVID-19. ${ }^{4-8}$ The condition of T2D has been identified to be an independent risk factor for predicting COVID-19 severity. ${ }^{7}$ Nevertheless, the severity of diabetes has not been fully considered in the quantitative assessment of critical care for COVID19. The outcome of COVID-19 might differ significantly in the T2D population with different level of glycemic control. Considering that the hospital and intensive care units (ICUs) are at full capacity/overcrowded with COVID-19 cases during the pandemic, ${ }^{9}$ identification of such a highrisk T2D cohort at the time of admission 
may allow for proper monitoring and management of subsequent care.

Several mechanisms linking glycemic control in T2D with COVID-19 progression have been suggested. First, inadequate glycemic control was shown to result in an impairment of immune response to infection in people with T2D, based on alteration in both cytokine profile and immune responses including T-cell and macrophage activation. ${ }^{10}$ Next, driven by T2D hyperglycemia, the excessive accumulation of advanced glycation endproducts (AGEs) from non-enzymatic glycation could lead to a loss of lung function and respiratory efficiency, through a reduction in lung muscle strength and elastic recoil capacity. ${ }^{11}$ Lastly, lack of glycemic control in T2D is often associated with chronic comorbidities such as cardiovascular diseases, obesity, and hypertension, all of which are known risk factors for COVID-19 infection and severity. ${ }^{8}$

Glycated hemoglobin (HbA1c) is a serum-based glycemic marker routinely measured in people with T2D, which captures average blood glucose levels over the duration of 2-3 months. ${ }^{12}$ As non-enzymatic glycation is a systemic diffusion-based process which affects multiple organs for years, to better capture the severity and long-term effects of diabetes (and its chronic comorbidities), longitudinal HbAlc over a longer period may be more clinically relevant than plasma glucose level or single-point HbAlc measured at the time of admission. Previous research in our group demonstrated that the 2-year longitudinal HbAlc is significantly correlated to following 2-year bone fracture risk in people with T2D. ${ }^{13}$ Here, we posit that longitudinal HbA1c over 2years has a more significant relationship with COVID-19 severity among people with T2D, compared with plasma glucose level or single-point $\mathrm{HbAlc}$ at time of admission.

Proper management of glycemic control through antidiabetic treatment may reduce inflammation and relieve the burden of COVID-19 on respiratory systems. When diabetic individuals are infected with COVID-19, the diabetic treatment strategy may need to be modified amid potential adverse outcomes. ${ }^{14}$ With no known side effects on respiratory systems, insulin is generally recommended to treat critically ill patients with COVID-19. ${ }^{415}$ Metformin has multiple beneficial impacts targeting T2D, including optimizing glucose control, anti-inflammation, improving insulin resistance, and inhibiting pathways of immune hyperactivation, etc, which can also reduce the severe symptoms of COVID-19. ${ }^{16}$ Corticosteroids were found to be beneficial in treating patients with COVID-19 by decreasing inflammation and suppressing the overresponse of immune system to prevent organ damage. ${ }^{17}$ Specifically, three types of systemic corticosteroids (dexamethasone, hydrocortisone, and methylprednisolone) were shown to reduce mortality by $20 \%$ in critically ill patients with COVID-19. ${ }^{18}$ However, use of corticosteroids may exacerbate insulin sensitivity and glycemic control. ${ }^{19}$ Furthermore, antihypertensive therapies are widely used among diabetic population as hypertension is one of the major comorbidities of T2D. However, the use of antihypertensive treatments, such as ACE inhibitors (ACEIs) and angiotensin receptor blockers (ARBs), is still debated for treating COVID-19. These treatments could block angiotensin II signaling to reach therapeutic effects, but in the mean time, they increase ACE2 membrane activities which may worsen COVID-19 condition. ${ }^{20}$ There were also mixed outcomes in whether ACEIs/ARB therapy could reduce mortality in COVID-19. ${ }^{82} 22$ To properly manage diabetic condition in patients with COVID-19, there is an urgent need to examine the association of these common ongoing treatments at time of COVID-19 diagnosis with COVID-19-related severe diseases in a large T2D population-based database.

To this end, the objectives of this study are: (1) to determine whether 2 to 3-year longitudinal HbAlc (HbA1c aggregated from January 2017 to January 2020) is independently associated with COVID-19 severity and advances the risk assessment in people with T2D, and whether shorter or longer HbAlc aggregation presents more optimal association (from 3 to 6-month period prior to COVID-19 diagnosis, up to HbAlc aggregated from January 2016 to January 2020); (2) to investigate the short-term associations between common treatments for individuals with T2D and the risk of becoming critically ill.

\section{RESEARCH DESIGN AND METHODS}

\section{Data collection}

This study used deidentified administrative claims and electronic health record (EHR) data with linked laboratory results from the OptumLabs Data Warehouse (OLDW). The database contains longitudinal health information on enrollees and patients, representing a diverse mixture of ages, ethnicities and geographical regions across the USA. ${ }^{23}$ The claims data in OLDW include medical and pharmacy claims, laboratory results and enrollment records for commercial and Medicare Advantage enrollees. The EHR information is sourced from a multitude of EHR software. Data are collected from a variety of provider systems including integrated delivery networks, as well as stand-alone hospitals, inpatient and outpatient clinics, laboratories, skilled nursing facilities, and other healthcare providers. ${ }^{24}$ The EHRderived data include a subset of EHR data available from the commercial and Medicare Advantage enrollees that has been normalized and standardized into a single database. ${ }^{23}$

\section{Study population}

The study cohort is illustrated in figure 1 . The retrospective cohort study was conducted using data during the period of 1 January 2017 to 6 November 2020. The end of study period was selected 3 months prior to the time during which this study was conducted, as there is a recommended 6-month lag for the administrative claims data to ensure claims are completely adjudicated. First, 

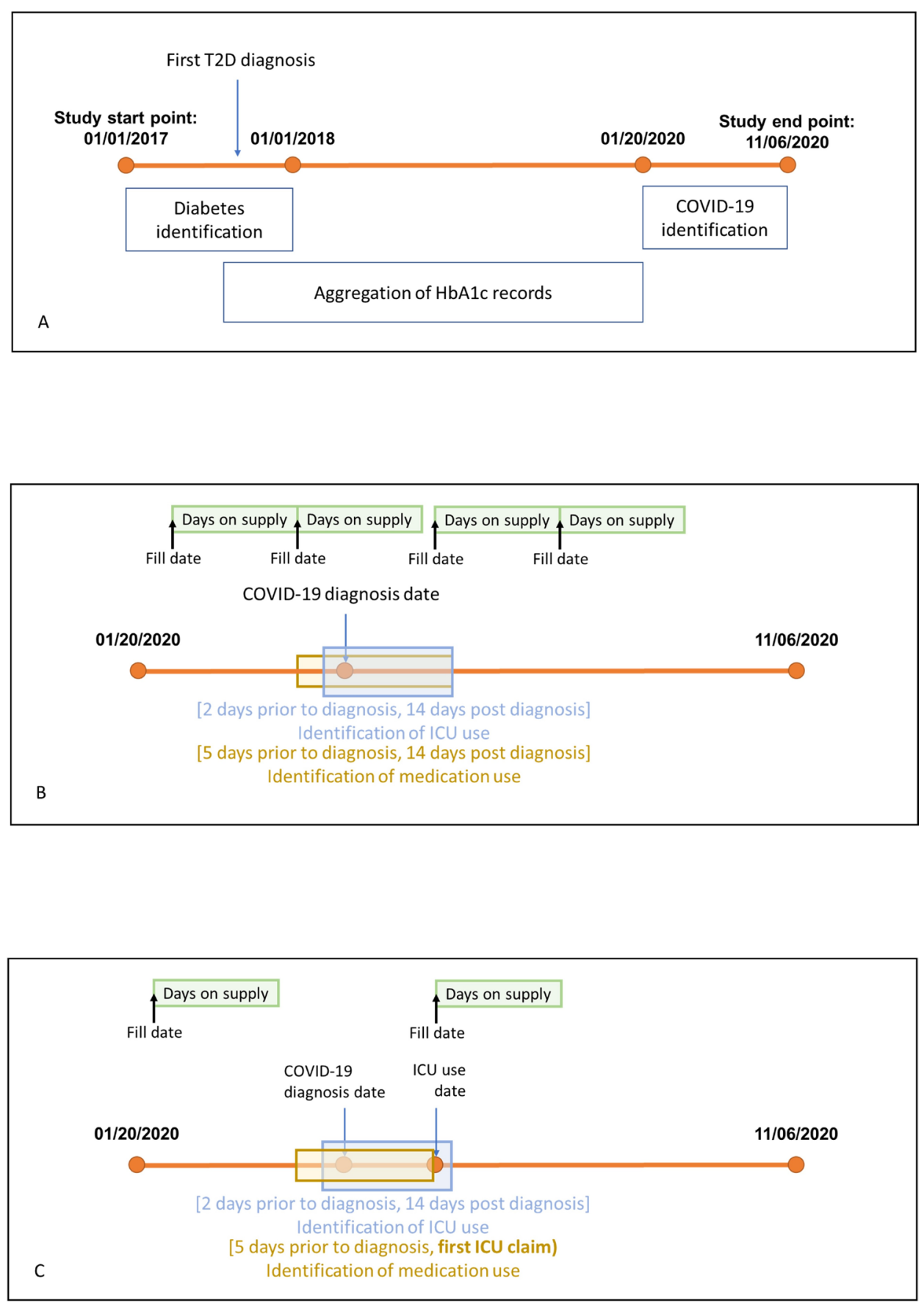

Figure 1 (A) The illustration of the study period. (B) An example of determining medication use during the COVID-19 window when no intensive care unit (ICU) claim was identified. Here, the medication supply period (green bar) is overlapping with medication identification period (yellow bar). Therefore, it is considered that the certain medication was used to treat COVID-19. (C) An example of determining medication use during the COVID-19 window when ICU claim(s) was identified. Instead of 2 weeks after COVID-19 first diagnosis, the end of medication identification window was defined as the day before first ICU claim. Here, since there was no overlapping between the medication supply period (green bar) and medication identification period (yellow bar), it is considered that this medication was not used to treat COVID-19. HbA1c, glycated hemoglobin; T2D, type 2 diabetes.

from 1 January 2017 to 1 January 2018, individuals with at least one T2D diagnosis claim were identified using International Classification of Diseases, Tenth Revision (ICD10), Clinical Modification codes. For each individual, the date of first diagnosis claims was defined as their index date. Starting from 15 days prior to their index date to 20 January 2020, each individual's HbA1c test results were collected from both claims linked lab results and EHR data sets. If the individual had more than one HbAlc test result, the multiple values were averaged. The individual's last HbA1c entry since 1 January 2019 to 20 January 2020 (if applicable) was also extracted to compare between single-point and longitudinal average of HbAlc. Several selection criteria were applied to the population: 
(1) age $\geq 18$ years old, with known sex; (2) continuously enrolled in Medicare/commercial coverage from the index date to the end of study period; (3) at least two inpatient or outpatient visits confirming the diabetic status within 1 year following the index date; (4) during the period of HbAlc aggregation, at least one HbAlc record is available. Only individuals with average HbAlc $\geq 6 \%$ were considered in the study. ${ }^{12}$ Considering that for majority of people with diabetes, the first $\mathrm{HbAlc}$ entry for this study would be recorded at their index date, this requirement usually warrants one test result existing for more than 2 years. Among the selected population, the individuals who had at least one positive COVID-19 diagnosis claim from 21 January 2020 to 6 November 2020 were extracted for the final study cohort. Individuals who did not meet the above selection criteria and/or had missing information were excluded. A total of 16504 participants, attritted from 2343169 T2D enrollees, were included in the study. People who had one or more positive COVID-19 diagnosis claims but no diabetic diagnosis claim during the entire study period were grouped into a non-diabetic control group for comparison $(n=379701)$.

Using the selected population, we constructed three study cohorts to compare if shorter or longer period of HbA1c aggregation was associated with COVID-19related severity. The first two cohorts were constructed using the T2D population between 1 January 2016 and 1 January 2017 ( $\mathrm{n}=21394)$, and between 1 January 2018 and 1 January 2019 ( $\mathrm{n}=15624)$, with other selection criteria remaining the same. In this manner, compared with 2 to 3-year HbAlc aggregation, we can determine whether a longer period (ie, 3-4 years, HbAlc aggregated from 1 January 2016 to 20 January 2020) or a short period (ie, 1-2years, HbA1c aggregated from 1 January 2018 to 20 January 2020) could affect the outcome. The third study cohort was constructed using the selected population between 1 January 2017 and 1 January 2018 where individual HbAlc values were measured over 3 to 6-month period prior to COVID-19 diagnosis $(\mathrm{n}=2040)$. This cohort allowed comparing the value within the period close to admission in predicting COVID-19related severity over previous two cohorts (that included HbA1c aggregation over shorter or longer periods). Other requirements were unchanged.

\section{Exposure and outcome definition}

The aggregated longitudinal $\mathrm{HbAlc}$ values for each individual were binned in two ways: (1) by $1 \%$ difference $(6 \%-7 \%, 7 \%-8 \%, 8 \%-9 \%, 9 \%-10 \%, 10 \%-11 \%, \geq 12 \%)$, and (2) by adequate glycemic control (longitudinal HbA1c 6\%-9\%) and poor glycemic control (longitudinal HbAlc $\geq 9 \%$ ). Currently, there is no global standard for quantification of glycemic control using HbAlc. Here, we selected the standard for poor glycemic control based on previous studies. ${ }^{25-27}$ To provide further information regarding glycemic control, other binning methods for poor glycemic control (longitudinal HbAlc $\geq 7 \%$ and longitudinal HbA1c $\geq 8 \%$ ) were also analyzed. During the same period where HbAlc values were collected (between 15 days prior to individual's index date and 20 January 2020), the diagnosis claims for common diabetic comorbidities were identified using ICD-10 codes. The selected comorbidities include hypertension, neuropathy, retinopathy, stroke, nephropathy, coronary artery disease (CAD), and obesity. The ICD-10 codes for diagnosis identification are listed in online supplemental table S1. Between 2 days before and 14 days after the individual's first COVID-19 diagnosis claim, the ICU service claims were extracted as an indication of patient developing COVID-19 severity. It is worth noting that the revenue codes used for ICU claims identification with description of surgical, pediatric, psychiatric, burn care, and trauma were excluded. For the study cohort, the fill date, and days of supply of five types of common medication use: metformin, insulin, ACEIs, ARBs, and corticosteroids, were recorded during the year 2020. The generics of medications extracted are listed in online supplemental table S2. Medication use was determined for the period between 5 days prior and 2 weeks after their first COVID-19 diagnosis. If the patient were ever admitted to ICU, an extra requirement for medications was set where the medication continuation had to be before the patient's first ICU claim to avoid collider bias (ie, if the individual only had medication fills after the first ICU claim, the medication records would not be considered). For the use of glucose-lowering agents (metformin and insulin), the association was examined both for their individual use and combined use. Considering the period for determination of medication use was relatively short, we assumed that the medication use continued for the entire window.

\section{Statistical analyses}

Kaplan-Meier survival model was used to estimate the unadjusted instantaneous risk of developing COVID-19 severity (defined by risk of ICU use), adequate glycemic control, and poor glycemic control groups. Log-rank test was used to quantify whether the risks between the groups were statistically different. Moreover, Cox proportional hazards model as a multivariate regression model was used to assess the independent HRs of longitudinal $\mathrm{HbA1c}$, as well as various medications, when adjusting for other confounding factors such as age, sex, and diabetic comorbidities. HRs with $95 \%$ CI were calculated for each variable while accounting for all other variables. For all statistical tests, a $\mathrm{p}$ value $<0.05$ was considered significant. The same statistical models were applied when the individuals' last HbAlc record since 2019 was used instead of their two-year average, and for all three cohorts mentioned above in order to compare between single-point $\mathrm{HbAlc}$ and different periods of longitudinal HbA1c in relation to COVID-19-related severity. Data extraction and attrition were conducted using DbVisualizer software (DbVis Software, Stockholm, Sweden). All statistical analyses were performed in Python. 
Table 1 Demographics

\begin{tabular}{|c|c|c|c|c|c|}
\hline \multicolumn{6}{|c|}{ Study cohort, $n=16504$} \\
\hline \multirow[t]{2}{*}{ Age } & Mean & SD & & & \\
\hline & 67.6 & 12.0 & & & \\
\hline Sex & $\mathbf{n}$ & $\%$ & Medications used & $\mathbf{n}$ & $\%$ \\
\hline Male & 7865 & 47.7 & Metformin & 6504 & 39.4 \\
\hline Female & 8639 & 52.3 & Insulin & 3889 & 23.5 \\
\hline Race & $\mathbf{n}$ & $\%$ & ACEls & 4261 & 25.8 \\
\hline Asian & 526 & 3.2 & ARBs & 4039 & 24.5 \\
\hline Black & 3490 & 21.1 & Corticosteroids & 1085 & 6.6 \\
\hline Hispanic & 3687 & 22.3 & Comorbidities & $\mathbf{n}$ & $\%$ \\
\hline White & 7589 & 46.0 & Neuropathy & 8074 & 48.9 \\
\hline Other/unknown & 1212 & 7.3 & Retinopathy & 6735 & 40.8 \\
\hline HbA1c bins & $\mathbf{n}$ & $\%$ & Nephropathy & 8567 & 51.9 \\
\hline $6 \%-7 \%$ & 7212 & 43.7 & Coronary artery disease & 6449 & 39.1 \\
\hline $7 \%-8 \%$ & 4466 & 27.1 & Stroke & 2302 & 13.9 \\
\hline $8 \%-9 \%$ & 2360 & 14.3 & Hypertension & 15614 & 94.6 \\
\hline $9 \%-10 \%$ & 1215 & 7.4 & Obesity & 8797 & 53.3 \\
\hline $10 \%-11 \%$ & 623 & 3.8 & Severity & $\mathbf{n}$ & $\%$ \\
\hline $11 \%-12 \%$ & 347 & 2.1 & ICU usage & 2952 & 17.9 \\
\hline $12 \%+$ & 281 & 1.7 & & & \\
\hline
\end{tabular}

ACEI, ACE inhibitor; ARB, angiotensin receptor blocker; ICU, intensive care unit.

\section{RESULTS}

From the database, we initially identified 2343169 individuals with T2D diagnosis claims. Following the data filtering based on the criteria described in the Research design and methods section, 1428292 people with diabetes were selected. Among this population, 16504 people who had at least one claim for positive COVID-19 diagnosis before 6 November 2020 were defined as our study cohort. The demographics of the study cohort are described in table 1. During the HbAlc aggregation period, the mean HbAlc frequency was 4.7. The frequency for HbAlc measurement is included in online supplemental table S3. In this cohort, 2952 (17.9\%) patients had at least one claim for ICU usage between 2 days before and 14 days after their initial COVID-19 diagnosis.

We used Kaplan-Meier survival model as a univariate model to estimate the COVID-19 severity risk defined by the risk of ICU usage in people with diabetes. The study cohort was divided into an adequate glycemic control group $(\mathrm{n}=14038)$ and a poor glycemic control group $(\mathrm{n}=2466)$. The non-diabetic individuals infected with COVID-19 were grouped for comparison $(\mathrm{n}=379701)$. Figure 2 shows the risk estimation of three groups described above. Log-rank test determined that differences in risk of ICU usage between all three groups are statistically significant (all $\mathrm{p}<0.001)$.

Next, we used Cox proportional hazards model to investigate the independent instantaneous risk of ICU usage by longitudinal HbAlc, as well as medications commonly used for people with T2D when adjusted for other confounding factors. Figure 3 listed the HRs of each variable. The HR of 2 to 3-year longitudinal HbAlc (stratified by adequate and poor glycemic control $(9 \%$ cut-off) ) was 1.48 (95\% CI 1.34 to 1.63 , p<0.001), which is shown to be the largest independent risk factor for intensive care. By changing the poor glycemic control cut-off to $\geq 8 \%$ and $\geq 7 \%$, the $\mathrm{HR}$ of longitudinal $\mathrm{HbA} 1 \mathrm{c}$ reduced to $1.34(95 \%$ CI 1.23 to $1.45, \mathrm{p}<0.001)$ and $1.21(95 \%$ CI 1.12 to $1.31, \mathrm{p}<0.001)$, respectively. When binary binning was changed to per $1 \%$ difference, the HR of longitudinal HbA1c was 1.12 (95\% CI 1.09 to $1.15, \mathrm{p}<0.001)$. This finding represents that $1 \%$ increase in longitudinal HbA1c is a directly associated $12 \%$ increase in the risk of ICU use.

Conversely, when the variable of longitudinal HbAlc was changed to their last HbAlc entry (single point), the HRs of HbA1c were reduced to 1.36 (adequate or poor glycemic control, 95\% CI 1.22 to $1.53, \mathrm{p}<0.001)$ and 1.10 (per $1 \%$ increase, $95 \%$ CI 1.07 to $1.13, \mathrm{p}<0.001$ ). When the HbAlc values were aggregated for the 3 to 6 -month period prior to COVID-19 diagnosis, the risk of ICU use was not significantly different between poor and adequate glycemic control groups in the univariate model $(\mathrm{p}=0.23)$ and $\mathrm{HbAlc}$ was not a significant covariate in the Cox proportional hazards model (HR: $1.27,95 \%$ CI 0.98 to $1.64, \mathrm{p}=0.07)$. When longitudinal HbAlc was aggregated only for 1-2years, people with poor glycemic control 


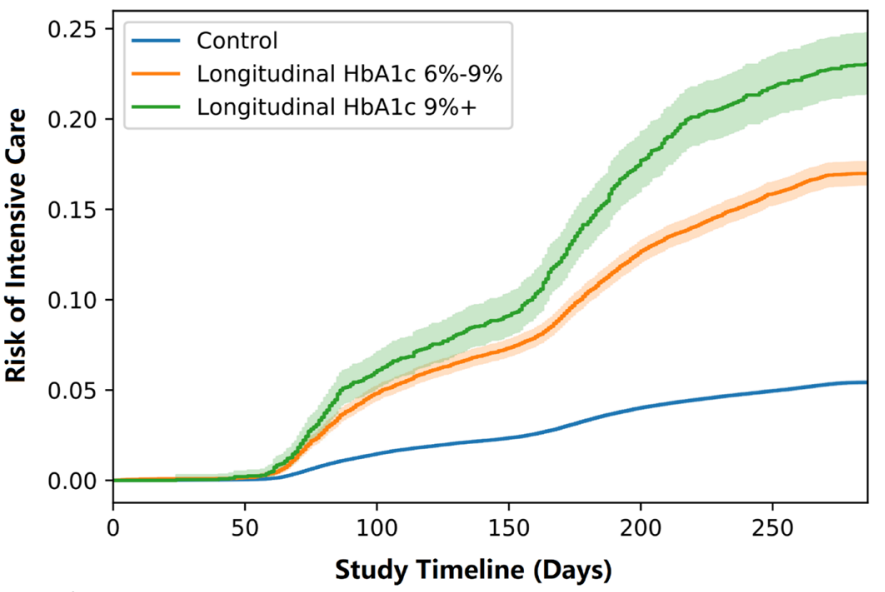

\begin{tabular}{|c|c|c|c|c|c|c|c|}
\hline \multirow[b]{2}{*}{ Non-diabetic control } & \multicolumn{6}{|c|}{ Number of people who were admitted into ICU in each time point } & \multirow{2}{*}{$\begin{array}{l}\text { Censored counts } \\
358896\end{array}$} \\
\hline & 0 & 151 & 5489 & 8853 & 15163 & 18768 & \\
\hline Longitudinal $\mathrm{HbA} 1 \mathrm{c} 6 \%-9 \%$ & 0 & 21 & 672 & 1025 & 1766 & 2223 & 11654 \\
\hline Longitudinal HbA1c $9 \%+$ & 0 & 5 & 147 & 224 & 430 & 536 & 1898 \\
\hline
\end{tabular}

Figure 2 Kaplan-Meier survival model estimating the risk of intensive care unit (ICU) usage in patients with diabetes infected with COVID-19 from 21 January to 6 November. The risk of ICU usage was stratified by adequate glycemic control (longitudinal HbA1c 6\%-9\%, $n=14038$ ) and poor glycemic control (longitudinal HbA1c higher than 9\%, $n=2466$ ), shown with $95 \% \mathrm{Cl}$. A non-diabetic control group was added for comparison $(n=379701)$.

show $42 \%$ increased risk of ICU use (HR: $1.42,95 \%$ CI 1.30 to $1.56, \mathrm{p}<0.001)$. The 3 to 4 -year HbA1c aggregation demonstrated a similar HR to 2 to 3-year HbAlc aggregation, showing a $49 \%$ increased risk of ICU use for people with poor glycemic control (HR: $1.49,95 \%$ CI 1.34 to $1.65, \mathrm{p}<0.001)$.

For the two antidiabetic medications taken individually (ie, metformin use only, or insulin use only), metformin shows significant positive effects in reducing risk of ICU use by $12 \%$ (HR: $0.88,95 \%$ CI 0.81 to $0.97, p=0.01$ ) while the influence of insulin use is not significant. The combined use of the two antidiabetic medications shows an $18 \%$ decrease in the ICU use risk (HR: $0.82,95 \%$ CI 0.71 to 0.95 , $\mathrm{p}=0.01$ ). Use of corticosteroids was related to a reduction in the ICU use risk by $29 \%$ (HR: $0.71,95 \%$ CI 0.60 to 0.84 , $\mathrm{p}<0.001)$. In contrast, the two classes of antihypertensive medications, ACEIs and ARBs, were not associated with a decrease in the ICU use risk. We found that ARBs were significantly correlated to an increased ICU usage (HR: $1.17,95 \%$ CI 1.07 to $1.28, \mathrm{p}<0.005)$ even after the coronary artery conditions were adjusted. Sex and age also influence COVID-19 severity risk. The risk of using ICU was 30\% higher in males than in females. For every 1-year increase in age, the risk of using ICU is increased by $1 \%$. Among the common diabetic comorbidities, hypertension, nephropathy, neuropathy, and obesity significantly increased the risk of ICU use. Stroke, CAD, and retinopathy did not show a significant impact on ICU usage.

\section{CONCLUSIONS}

Given the situation, when hospitals and ICUs are at full capacity/overcrowded with COVID-19 cases, it is

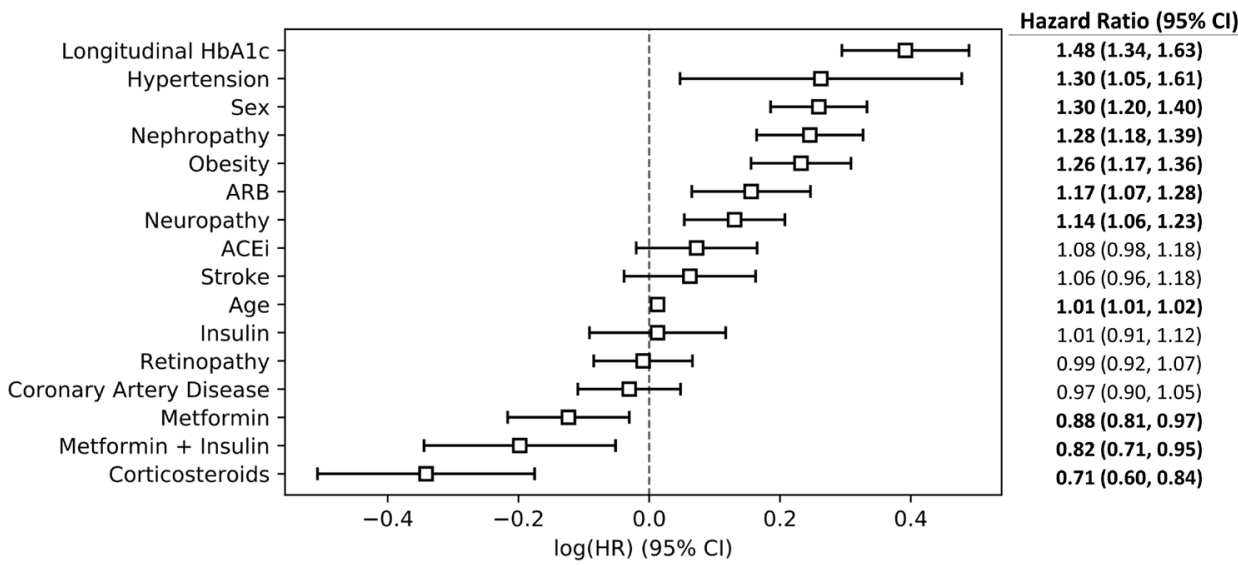

Figure 3 Cox proportional hazards model estimating the HR of each variable while accounting for all other variables. The statistically significant HRs are in boldface. Here, longitudinal HbA1c, which is stratified by adequate and poor glycemic control, is shown to be the largest risk factor for COVID-19-related severity. When longitudinal HbA1c is categorized per $1 \%$ difference, its HR is 1.12 (95\% Cl 1.09 to 1.15, p<0.001). ACEi, ACE inhibitor; ARB, angiotensin receptor blocker. 
imperative to identify ahead of time (at the time of admission using existing records) individuals at risk of developing severe diseases and potentially in need of intensive care, especially for T2D population that is disproportionally affected by COVID-19. ${ }^{4-8}$ Here, we have discovered the remarkable impact of 2 to 3-year longitudinal glycemic control on the COVID-19-related severity in a cohort containing 16504 patients with COVID-19 selected from more than 2.3 million people with T2D listed in a US population-based data set with a broad distribution of geographical regions and ethnicities. Longitudinal HbA1c, a simple, universal, and cost-effective measurement with a reasonable link to the pathophysiology of COVID-19 progression, can be used to quantitatively assess the risk of intensive care in the general T2D population at the time of admission to allow proper monitoring and management of subsequent care. Among the common medications for people with T2D, we found that the combined use of glucose-lowering agents as well as corticosteroids was associated with a decreased risk of COVID-19 severity, while the antihypertensive medications either had no significant effect or were associated with an increase in severity risk. Furthermore, consistent with previous investigations, ${ }^{8}$ we find that three types of common diabetic comorbidities including hypertension, nephropathy, and obesity increase the COVID-19 severity in patients with T2D.

It is noteworthy that inadequate glycemic control over time could also lead to the development of the diabetic comorbidities noted above. ${ }^{25} 28$ Previously, we have identified that the occurrence rate of several diabetic comorbidities is significantly correlated to the 2-year longitudinal HbAlc. ${ }^{13}$ As quantification of the duration associated with these comorbidities and the severity of the comorbidities continues to be challenging, longitudinal HbAlc could account for the increased risk of COVID-19 severity caused by comorbidities. Additionally, our findings indicate that the gender and age-related differences in COVID-19 severity are comparable among T2D population and general population. ${ }^{29} 30$

Demonstrated through the Kaplan-Meier survival model, we initially estimated the unadjusted risk of ICU usage solely stratified by diabetes status and longitudinal HbA1c levels. By the end of the study period, there was a pronounced increase in ICU use risk in T2D population than non-diabetic controls. The elevated risk of ICU use was clearly distinguishable in people with poor glycemic control compared with those with adequate glycemic control. The value and utility of 2 to 3-year longitudinal HbAlc was further confirmed in the Cox proportional hazards model where other potential risk factors were adjusted. Grouping the cohort by adequate and poor glycemic control, longitudinal HbAlc level was shown to be the largest independent risk factor in the model. Previous studies have investigated the relationship between blood glucose and HbAlc with COVID-19. The results show that a single-point HbAlc at time of admission was either not related to COVID-19 outcomes $^{21} 3132$ or it was associated with increased COVID-19-related mortality. ${ }^{8}$ However, one study did indicate a significant positive association between singlepoint preinfection $\mathrm{HbAlc}$, measured within 6 months, and the severity of COVID-19 illness. ${ }^{33}$ In contrast to these previous investigations, our study considers a longer period glycemic control for an individual as an indicator for history of diabetes and severity (which are usually harder to evaluate) by using the 2 to 3 -year longitudinal HbA1c. Here we successfully identified the 2 to 3-year longitudinal HbA1c to be a significant risk factor for COVID-19-related severity that can serve as a key clinical parameter related to management of COVID-19 for an individual patient and for assessing ICU use. Furthermore, longitudinal HbAlc is physiologically more relevant because it captures hyperglycemia over an extended period and hyperglycemia leads to accumulation of AGEs through the diffusion-driven process of non-enzymatic glycation. Also the excessive accumulation of AGEs could indeed result in the loss of lung function and respiratory efficiency through a reduction in lung muscle strength and elastic recoil capacity. ${ }^{11}$

Although single-point HbA1c (last record since 2019) was shown to be a significant factor related to the risk of ICU use in our statistical model, its relatively low HR, when compared with longitudinal HbAlc, shows that the risk of ICU use is underestimated by such an association. In contrast, longitudinal HbA1c average presents a more significant and evident association. Our study demonstrates the different associations with risk of intensive care when different HbAlc aggregation periods are selected. It was shown that the longer the period over which HbA1c is aggregated, the stronger its association with COVID-19related severity is. Furthermore, when HbAlc was only aggregated close to the admission date (3-6 months prior to COVID-19 diagnosis), the relationship between HbA1c and COVID-19-related severity was not significant. Although this lack of significance could be attributed to the use of a small sample $(n=2040)$ compared with the full original cohort $(n=16504)$, aggregating HbA1c in this smaller time window yielded the lowest $\mathrm{HR}$ of $\mathrm{HbA1c}$ (1.27) in comparison to the other groups. Conversely, 2 to 3-year HbAlc and 3 to 4-year HbAlc presented the highest HR. Since the increased risk by longitudinal HbA1c is essentially not different between these groups, it is suggested that a saturation of association might have been reached after aggregating the HbAlc values for 2-3years. Considering the difficulties of gathering HbA1c information for extended period, 2-3 years is a moderate time window which is sufficient for assessment of COVID-19-related subsequent care.

Four types of commonly prescribed medications for people with T2D (two glucose-lowering agents: metformin and insulin, two blood pressure-lowering agents: ACEIs and ARBs) were examined to determine whether their continued use interferes with COVID-19 progression. Corticosteroids, although imposing the risk of elevating blood glucose level, have shown beneficial 
effects in reducing COVID-19 mortality in the general population. The evaluation of all medications should be interpreted with caution since this study is not a randomly controlled trial. Insulin, when administered individually (ie, no metformin use), despite having an HR of 0.93 , did not demonstrate a significant effect. In contrast, consistent with previous studies, ${ }^{34}$ metformin individually was associated with significantly lower risk of ICU use by $12 \%$. It can be hypothesized that the beneficial effects of metformin besides glycemic control, such as anti-inflammation and inhibition of viral entry, could help prevent the patients with T2D from becoming critically ill, whereas the stronger glucose-lowering effect of insulin might be more crucial in patients with T2D who are already in a critical condition. It is worth noting that the metformin users potentially have a shorter history of diabetes and less severity in terms of diabetic comorbidities. That being said, the association of medications was adjusted based on their longitudinal HbAlc level and comorbidity conditions. The group with combined use of metformin and insulin displayed the best effect compared with the individual use, suggesting that more intensive glycemic control may further reduce the risk of COVID-19-related severity in people with T2D.

The effect of ACEIs was shown to be not significant. Interestingly, ARBs did associate with higher risk of ICU use. A previous observational study also reported that the antihypertensive medications are correlated with increased risk of COVID-19-related mortality in people with T2D. ${ }^{8}$ Besides the mechanism that antihypertensive medications could increase ACE2 expression on membrane which allows higher level of viral entry, this result is most likely due to confounding by indication. Although the associations of ACEIs and ARBs were adjusted for the conditions of hypertension and CAD, the systolic blood pressure at the time of COVID-19 diagnosis was not available for our analyses. Yet, others have shown that the negative effect of antihypertensive medications remains even after adjustment for systolic blood pressure. ${ }^{8}$ ARBs specifically were often used as a substitute for people more likely to develop angioedema from ACEIs. Angioedema has been hypothesized to favor COVID-19 progression, and in turn COVID-19 can further exacerbate pre-existing angioedema condition. ${ }^{35}$ Therefore, people with T2D who were using ARBs might essentially be at higher risk for developing critical conditions related to COVID-19.

The three generic forms of corticosteroids were found to be most beneficial among all selected medications in reducing the risk of ICU use by $28 \%$. This finding is similar to previously summarized association of corticosteroids and lower all-cause mortality in general population infected with COVID-19. ${ }^{36}$ Despite the observations that administration of corticosteroids could worsen glycemic control, ${ }^{19}$ our results suggest that previous or ongoing treatment of corticosteroids might still be effective in people with T2D. Notably, the number of users for the selected corticosteroids in this cohort is relatively small $(\mathrm{n}=662,5.4 \%)$. Among this group of people, about $65 \%$ were either on metformin or insulin. It is possible that the corticosteroid users in this cohort potentially had better glycemic control at the time around COVID-19 diagnosis. However, in the HbAlc aggregation window, the longitudinal HbAlc level between corticosteroid users (average longitudinal HbA1c: 7.5) and noncorticosteroid users (average longitudinal HbA1c: 7.6) was not much different. Altogether, the positive association between corticosteroids and COVID-19-related severity should be treated cautiously. The decision of using corticosteroids should be considered along with proper management of glycemic level and on a case-bycase basis.

Two limitations of the study should be noted. First, the study used the individual's average of 2 to 3-year HbAlc. Our results did show that the average value itself is sufficient to properly evaluate the risk of COVID-19-related severity. Nevertheless, the longitudinal trend and deviation of HbAlc level during the 2 to 3-year window could potentially play an additional role in the association and requires future investigations. Second, the medication use was based on the administrative claims data. It is rational to assume that the medication use is continued during the short identification window. However, the individuals' medication adherence could not be verified. The daily dosage information was also not included in the analysis.

In conclusion, here we identified that the 2 to 3-year longitudinal glycemic control is most significantly associated with COVID-19-related severity in people with T2D. Proper management of longer period glycemic level could potentially be essential in reducing the risk of developing severe diseases from COVID-19. This method using HbAlc history could also allow for personalized assessment and management of subsequent care related to COVID-19 in advance. Additionally, the combined use of metformin and insulin and the use of corticosteroids are effective to prevent patients with T2D from becoming critically ill from COVID-19.

\section{Author affiliations}

${ }^{1}$ Center for Biotechnology and Interdisciplinary Studies, Department of Biomedical Engineering, Rensselaer Polytechnic Institute, Troy, New York, USA

${ }^{2}$ OptumLabs Visiting Fellow, Optum Health, Eden Prairie, Minnesota, USA ${ }^{3}$ Department of Genetics and Genomic Sciences, Icahn School of Medicine at Mount Sinai, New York City, New York, USA

${ }^{4}$ Hasso Plattner Institute for Digital Health at Mount Sinai, Icahn School of Medicine at Mount Sinai, New York City, New York, USA

${ }^{5}$ Division of Nephrology, Department of Medicine, Icahn School of Medicine at Mount Sinai, New York City, New York, USA

Acknowledgements We would like to thank Tanya Natwick, Laura Becker, and Donna Spencer of OptumLabs for providing support in data set construction.

Contributors DV is the principal investigator of the study. BSG and GNN are coinvestigators. All authors contributed to study design. BW contributed to data collection and organization. Data were analyzed by BW, and interpreted by BW, BSG, GNN and DV. BW drafted the manuscript and revisions were suggested by DV, BSG, and GNN. The final version was approved by all the authors. BW will take responsibility for integrity of data analyses. 
Funding This work was supported by the National Institutes of Health's (NIH) National Institute of Arthritis and Musculoskeletal and Skin Diseases (NIAMS) (R21AR071681).

\section{Competing interests None declared.}

Patient consent for publication Not required.

Ethics approval This study involves human participants, but the Institutional Review Board at Rensselaer Polytechnic Institute exempted this study. Patient identifiers have been coded or removed prior to its release to the study investigators, such that it is compliant with HIPAA and exempt from Institutional Review Board review.

Provenance and peer review Not commissioned; externally peer reviewed.

Data availability statement Data may be obtained from a third party and are not publicly available. The data that support the findings of this study are available from OptumLabs Data Warehouse. Restrictions apply to the availability of these data, which were used under license for this study.

Supplemental material This content has been supplied by the author(s). It has not been vetted by BMJ Publishing Group Limited (BMJ) and may not have been peer-reviewed. Any opinions or recommendations discussed are solely those of the author(s) and are not endorsed by BMJ. BMJ disclaims all liability and responsibility arising from any reliance placed on the content. Where the content includes any translated material, BMJ does not warrant the accuracy and reliability of the translations (including but not limited to local regulations, clinical guidelines, terminology, drug names and drug dosages), and is not responsible for any error and/or omissions arising from translation and adaptation or otherwise.

Open access This is an open access article distributed in accordance with the Creative Commons Attribution Non Commercial (CC BY-NC 4.0) license, which permits others to distribute, remix, adapt, build upon this work non-commercially, and license their derivative works on different terms, provided the original work is properly cited, appropriate credit is given, any changes made indicated, and the use is non-commercial. See: http://creativecommons.org/licenses/by-nc/4.0/.

ORCID iD

Bowen Wang http://orcid.org/0000-0003-0049-1623

\section{REFERENCES}

1 WHO. WHO coronavirus (COVID-19) dashboard, 2021. Available: https://covid19.who.int

2 Ritchie H, Ortiz-Ospina E, Beltekian D. Coronavirus pandemic (COVID-19). Our World Data, 2020. Available: https://ourworldindata. org/covid-vaccinations

3 Lopez Bernal J, Andrews N, Gower C, et al. Effectiveness of Covid-19 vaccines against the B.1.617.2 (delta) variant. $N$ Engl $J$ Med 2021;385:585-94.

4 Apicella M, Campopiano MC, Mantuano M, et al. COVID-19 in people with diabetes: understanding the reasons for worse outcomes. Lancet Diabetes Endocrinol 2020;8:782-92.

5 Yang X, Yu Y, Xu J, et al. Clinical course and outcomes of critically ill patients with SARS-CoV-2 pneumonia in Wuhan, China: a singlecentered, retrospective, observational study. Lancet Respir Med 2020;8:475-81.

6 Zhang J-J, Dong X, Cao Y-Y, et al. Clinical characteristics of 140 patients infected with SARS-CoV-2 in Wuhan, China. Allergy 2020;75:1730-41

7 Liang X, Xu J, Xiao W, et al. The association of diabetes with COVID-19 disease severity: evidence from adjusted effect estimates. Hormones 2021;20:409-414.

8 Holman N, Knighton P, Kar P, et al. Risk factors for COVID-19-related mortality in people with type 1 and type 2 diabetes in England: a population-based cohort study. Lancet Diabetes Endocrinol 2020;8:823-33.

9 Conlen M, Keefe J, Leatherby L, et al. How full are hospital I.C.U.s near you? The New York Times, 2020. Available: https://www. nytimes.com/interactive/2020/us/covid-hospitals-near-you.html

10 Ferlita S, Yegiazaryan A, Noori N, et al. Type 2 Diabetes Mellitus and Altered Immune System Leading to Susceptibility to Pathogens, Especially Mycobacterium tuberculosis. J Clin Med 2019;8. doi:10.3390/jcm8122219. [Epub ahead of print: 1612 2019].

11 Ardigo D, Valtuena S, Zavaroni I, et al. Pulmonary complications in diabetes mellitus: the role of glycemic control. Curr Drug Targets Inflamm Allergy 2004;3:455-8.
12 American Diabetes Association. Diagnosis and classification of diabetes mellitus. Diabetes Care 2012;35 Suppl 1:S64-71.

13 Wang B, Wang Z, Poundarik A. Fracture risk in type 2 diabetes: longitudinal glycemic control based prediction and the efficacy of medications. Journal of Bone and Mineral Research 2020;35:30-1.

14 Papadokostaki E, Tentolouris N, Liberopoulos E. COVID-19 and diabetes: what does the clinician need to know? Prim Care Diabetes 2020;14:558-63.

15 Bouhanick B, Cracowski J-L, Diabetes FJ-L. And COVID-19. Therapies 2020;75:327-33.

16 Scheen AJ, Metformin SAJ. Metformin and COVID-19: from cellular mechanisms to reduced mortality. Diabetes Metab 2020;46:423-426.

17 Cano EJ, Fonseca Fuentes X, Corsini Campioli C, et al. Impact of corticosteroids in coronavirus disease 2019 outcomes: systematic review and meta-analysis. Chest 2021;159:1019-40.

18 Sterne JAC, Diaz J, Villar J, et al. Corticosteroid therapy for critically ill patients with COVID-19: a structured summary of a study protocol for a prospective meta-analysis of randomized trials. Trials 2020;21:734.

19 Egbuonu F, Antonio FA, Edavalath M. Effect of inhaled corticosteroids on glycemic status. Open Respir Med $J$ 2014;8:101-5.

20 Onweni CL, Zhang YS, Caulfield T, et al. ACEI/ARB therapy in COVID-19: the double-edged sword of ACE2 and SARS-CoV-2 viral docking. Crit Care 2020;24:475.

21 Chen Y, Yang D, Cheng B, et al. Clinical characteristics and outcomes of patients with diabetes and COVID-19 in association with glucose-lowering medication. Diabetes Care 2020;43:1399-407.

22 Zhang P, Zhu L, Cai J, Peng Z, Lihua Z, Jingjing C, et al. Association of inpatient use of angiotensin-converting enzyme inhibitors and angiotensin II receptor blockers with mortality among patients with hypertension hospitalized with COVID-19. Circ Res 2020;126:1671-81.

23 OptumLabs. OptumLabs and OptumLabs data warehouse (OLDW) descriptions and citation. PDF. Reproduced with permission from OptumLabs. Eden prairie, MN: OptumLabs, 2020.

24 Hang L. Panther user guide: data overview and use in applied research. n.p.. PDF. Reproduced with permission from OptumLabs. Cambridge, MA: OptumLabs, 2021

25 Juarez DT, Sentell T, Tokumaru S, et al. Factors associated with poor glycemic control or wide glycemic variability among diabetes patients in Hawaii, 2006-2009. Prev Chronic Dis 2012;9:120065.

26 Feldman BS, Cohen-Stavi CJ, Leibowitz M, et al. Defining the role of medication adherence in poor glycemic control among a general adult population with diabetes. PLoS One 2014;9:e108145.

27 Chiang CY, Bai KJ, Lin HH, et al. The influence of diabetes, glycemic control, and diabetes-related comorbidities on pulmonary tuberculosis. PLoS One 2015;10:e0121698.

28 Luo M, Lim WY, Tan CS, et al. Longitudinal trends in $\mathrm{HbA} 1 \mathrm{c}$ and associations with comorbidity and all-cause mortality in Asian patients with type 2 diabetes: a cohort study. Diabetes Res Clin Pract 2017;133:69-77.

29 Pradhan A, Olsson P-E. Sex differences in severity and mortality from COVID-19: are males more vulnerable? Biol Sex Differ 2020;11:53.

30 Jin J-M, Bai P, He W, et al. Gender differences in patients with COVID-19: focus on severity and mortality. Front Public Health 2020;8.

31 Zhu L, She Z-G, Cheng X, et al. Association of blood glucose control and outcomes in patients with COVID-19 and pre-existing type 2 diabetes. Cell Metab 2020;31:1068-77.

32 Cariou B, Hadjadj S, Wargny M, et al. Phenotypic characteristics and prognosis of inpatients with COVID-19 and diabetes: the CORONADO study. Diabetologia 2020;63:1500-15.

33 Hayek S, Ben-Shlomo Y, Balicer R, et al. Preinfection glycaemic control and disease severity among patients with type 2 diabetes and COVID-19: a retrospective, cohort study. Diabetes Obes Metab 2021;23:1995-2000.

34 Crouse A, Grimes T, Li P, et al. Metformin use is associated with reduced mortality in a diverse population with COVID-19 and diabetes. medRxiv 2020. doi:10.1101/2020.07.29.20164020. [Epub ahead of print: $31 \mathrm{Jul} 2020]$

$35 \mathrm{Xu}$ Y, Liu S, Zhang Y, et al. Does hereditary angioedema make COVID-19 worse? World Allergy Organ J 2020;13:100454

36 WHO Rapid Evidence Appraisal for COVID-19 Therapies (REACT) Working Group, Sterne JAC, Murthy S, et al. Association between administration of systemic corticosteroids and mortality among critically ill patients with COVID-19: a meta-analysis. JAMA 2020;324:1330-41. 